\title{
La Costituzione di Querètaro tra diritti fondamentali e principi di costituzione economica
}

\section{Marcello SALERNO*}

\author{
SOMMARIO: I. La tutela multilivello dei diritti. II. L'impronta \\ presidenzialista nell'organizzazione federale. III. Principi di \\ finanza pubblica e costituzione economica.
}

\section{LA TUTELA MULTILIVELLO DEI DIRITTI}

El estado de Querétaro es parte integrante de la Federación mexicana, es libre y autónomo en lo que se refiere a su régimen interno y sólo delega sus facultades en los poderes federales, en todo aquello que fije expresamente la Constitución Política de los Estados Unidos Mexicanos.

Con queste parole si apre la Costituzione di Querètaro, che si colloca nell'ordinamento federale in armonia con i principi costituzionali degli Stati Uniti del Messico. In questo paese, il federalismo è stato molto più che un sistema di distribuzione geografica delle competenze: "la sua unione iniziale con il liberalismo, il fatto di essere questo fonte, predecessore o parente, reale o artificiale, del progressismo e del rivoluzionarismo messicani, uniscono il federalismo al progresso e alla rivoluzione e lo op-

* Investigador en instituciones de derecho público en la Universidad "A. Moro" de Bari, Italia, y habilitado como profesor asociado. Correo electrónico: marcello.salerno@ uniba.it; ORCID: 0000-0003-0133-4806. 
pongono al centralismo, al conservatorismo e alla controrivoluzione"1. Il federalismo messicano, in altri termini, è strettamente correlato ai passaggi più importanti della storia costituzionale di questo paese. Nel contesto latino-americano, il Messico è stata la nazione nella quale l'organizzazione federale dello Stato è risultata più radicata. ${ }^{2}$ La vastità e la diversità del paese sono un dato strutturale che ha rappresentato fisiologicamente l'elemento idoneo a giustificare e radicare una soluzione di tipo federale.

La disciplina dei diritti fondamentali si inserisce organicamente all'interno del sistema federale con un modello di tutela multilivello. L'art. 2 della Costituzione di Querètaro afferma il riconoscimento dei diritti umani nel quadro dei diritti costituzionali degli Stati Uniti messicani, dei trattati internazionali di cui lo Stato messicano è parte e delle leggi federali. ${ }^{3}$ Il rinvio al sistema di garanzie sovrastatali, tuttavia, non esaurisce la disci-

1 García Ramírez, Sergio, "Prólogo. Sobre el federalismo mexicano", in Gamas Torruco, José (cur.), El federalismo mexicano, Messico, SepSetentas, 1975, pp. 10 e ss. ma cfr. anche Carpizo, Jorge, "Sistema federal mexicano", in id., Estudios constitucionales, Messico, 1983, p. 87. Il federalismo messicano è stato anche definito come "forza che attraversa la storia del Messico" o "dogma político constitucional", cfr. Fix-Zamudio, Héctor e Valencia Carmona, Salvador, Derecho constitucional mexicano y comparado, Messico, Porrúa, 2001, pp. 939 e 946. Il Messico, in effetti, costituisce uno dei più articolati e importanti esempi di Stato federale sin dal 1824, anno in cui fu adottata la prima Costituzione all'esito del difficile processo di conquista dell'indipendenza dalla Spagna. Da allora l'impronta federalista ha sostanzialmente caratterizzato l'ordinamento messicano fino a oggi (salvo la breve parentesi della Costituzione del 1843) e ne costituisce uno dei pilastri fondamentali: Ferndández Sesgado, Francisco, El federalismo en America latina, Siena, 2001; Carpizo, Jorge e Carbonell, Miguel, Diritto costituzionale messicano, Torino, 2010, p. 56.

2 Olivetti, Marco, "Il contesto attuale e l'inquadramento storico-Messico", in Bifulco, Raffele (cur.), Ordinamenti federali comparati, vol. II, Torino, Giappichelli, 2012, pp. 5 e ss.

3 Un quadro ricostruttivo in: Lara Ponte, Rodolfo, Los derechos humanos en el constitucionalismo mexicano, México, Porrúa, 1998; Carducci, Michele, Il difficile confronto tra Europa e America latina su diritto giurisprudenziale e tutela multiordinamentale dei diritti fondamentali, "Federalismi.it", n. 4, 2013, reperibile in http://www.federalismi.it/ focus/index_focus.cfm?FOCUS_ID=20, 2013, data di accesso 27 agosto 2018; Gambino, Silvio, "Multilevel constitutionalism e diritti fondamentali", Diritto pubblico comparato ed europeo, 2008, pp. 1144 e ss. Dall'art. 41 della Costituzione messicana si deduce anche la subordinazione delle Costituzioni statali a quella federale: in nessun caso le Costituzioni dei singoli Stati potranno contraddire le stipulazioni del "patto federale". Cfr. sul punto: Olivetti, Marco, "Autonomia costituzionale: procedimenti di approvazione, oggetti, garanzie - Messico”, in Bifulco, Raffaele (cur.), Ordinamenti federali comparati, cit., p. 106.

Esta obra está bajo una Licencia Creative Commons

Atribución-NoComercial-SinDerivar 4.0 Internacional, IIJ-UNAM. 
plina costituzionale dei diritti nello stato di Querètaro. La scelta del costituente, frequente ma non costante nelle Costituzioni statali messicane, è stata quella di dotarsi anche di un proprio catalogo di diritti che andasse ad integrare quello federale, anche al fine di affermare talune specificità dello Stato e, implicitamente, rafforzare l'identità territoriale nel contesto dell'ordinamento federale messicano. I numerosi interventi di riforma costituzionale che si sono succeduti negli ultimi anni (2008, 2011, 2013, 2016, 2017) hanno contribuito a "modernizzare" la disciplina dei diritti e a rendere la carta costituzionale di questo Stato più adeguata all'evoluzione del contesto sociale ed economico. Non essendo questa la sede per svolgere un'analisi approfondita e men che mai completa di tali diritti, la presente riflessione si soffermerà brevemente soprattutto su alcuni aspetti caratterizzanti la Costituzione di Querètaro, specie laddove sono rinvenibili elementi di originalità rispetto, in particolare, alla tradizione costituzionale europea.

Una prima parte dei diritti contenuti nella Costituzione statale si concentra sulla cura della persona e dei suoi bisogni primari, partendo dal diritto all'alimentazione "sufficiente e adeguata" sotto il profilo nutrizionale e in grado di garantire una vita salubre ed attiva ${ }^{4}$ (art. 3, Cost. stat.). Al fine di evitare malattie di origine alimentare, è previsto, altresì, l'impegno attivo dello Stato, chiamato ad attuare misure che promuovano l'acquisizione di buone abitudini alimentari tra la popolazione, promuovere la produzione e il consumo di alimenti ad alto valore nutritivo.

Tra i diritti fondamentali legati al benessere della persona si trova anche quello alla pratica sportiva, funzionale ad una migliore qualità della vita e allo sviluppo fisico. A tal fine, la Costituzione impone un ruolo attivo allo Stato e agli enti locali nella promozione e nell'organizzazione delle attività formative, ricreative e delle competizioni sportive. Sulla stessa linea

4 Per un quadro della problematica: Bottiglieri, Maria, "The Protection of the Right to Adequate Food in the Italian Constitution", Forum di Quaderni Costituzionali, Rassegna n. 11, 2015, traduzione in italiano: "La protezione del diritto al cibo adeguato nella Costituzione italiana", reperibile in http://www.forumcostituzionale.it/ wordpress/ wp-content/uploads/2006/12/bottiglieri.pdf; data di accesso 27-agosto-2018; Narula, Smita, "The right to food: holding global actors accountable under international law", Columbia Journal of Transnational Law, n. 44, 2006, pp. 691 e ss.; Gusmai, Antonio, "Il diritto fondamentale al cibo adeguato tra illusioni e realtà", Dirittifondamentali.it, n. 2, 2015, reperibile in http://www.dirittifondamentali.it/ fascicoli/anno-2015/numero-22015/ gusmai-il-diritto-fondamentale-al/, data di accesso 27 agosto 2018. 
si colloca il diritto all'ambiente sano (art. 5, Cost. stat.). Tale diritto, come è noto, si è affermato diffusamente in molti Stati europei durante il secolo scorso, spesso prima sul piano materiale e successivamente, in larga parte, anche sul piano formale. Il diritto all'ambiente, tuttavia, anche quando è inserito tra i diritti fondamentali, può essere efficacemente tutelato nella misura in cui trova il corretto bilanciamento con altri valori costituzionali quali, in particolare, quelli legati alla sfera delle libertà economica. Infatti, occorre onestamente ammettere che

...le istituzioni giuridiche dell'economia industriale non sono in grado di assumere drasticamente l'ambiente come valore sovraordinato e condizionante le esigenze produttive e distributive del mercato e che la più alta forma di tutela possibile e razionalmente dovuta è quella che tende a ridurre gli effetti aggressivi mediante una progressiva razionalizzazione del rapporto tra economia e ambiente, bilanciandone le esigenze secondo la "migliore tecnologia disponibile".

Particolarmente rigorose appaiono poi le dichiarazioni di principio sulla difesa della vita sin dal concepimento. Il diritto alla vita di ogni essere umano, infatti, viene riconosciuto sin dalla fecondazione, momento nel quale l'individuo viene considerato "nato" per tutti gli effetti giuridici, senza prevedere particolari forme di bilanciamento, ad esempio, tra diritti della madre e del concepito che invece si riscontrano diffusamente nelle legislazioni europee.

Notevoli elementi di modernità emergono con riferimento ai diritti alle nuove tecnologie. Il diritto ad Internet, ad esempio, assume le caratteristiche di un vero e proprio diritto sociale in quanto la Costituzione attribuisce allo Stato il compito di rendere effettivo tale diritto attraverso l'implementazione delle necessarie politiche ${ }^{6}$ (art. 6o., Cost. stat.). Particolare

5 Di Plinio, Giampiero, Principi di diritto ambientale, Milano, Giuffrè, 2008, pp. 16 e ss. il quale, nel teorizzare il concetto di "tutela bilanciata" o "comparativa", evidenzia come il diritto all'ambiente può realisticamente trovare applicazione nella sua accezione integrale ed "ecocentrica" solo in aree territorialmente limitate (aree naturali protette) dove è possibile introdurre un regime giuridico speciale nel quale gli interessi e le libertà economiche vengono subordinati al valore ambientale. Sul tema v. anche Caravita, Beniamino, Diritto dell'ambiente, Bologna, Il Mulino, 2005; Mezzetti, Luca (cur.), Manuale di diritto ambientale, Padova, Cedam, 2001.

6 Su tutti gli aspetti di questo nuovo diritto costituzionale v. Frosini, Tommaso Edoardo, "Il diritto di accesso a Internet", in Frosini, Tommaso Edoardo e Pollicino, Oreste Esta obra está bajo una Licencia Creative Commons Atribución-NoComercial-SinDerivar 4.0 Internacional, IIJ-UNAM. 
attenzione viene posta anche ai rischi delle nuove tecnologie informatiche il cui utilizzo, si afferma, deve garantire il rispetto dei diritti fondamentali delle persone.

Sotto il profilo del diritto all'istruzione, la Costituzione di Querètaro si concentra sulla scelta del modello scolastico, individuando anche i valori del sistema educativo statale: democrazia, legalità, rispetto dei diritti umani, lavoro e produttività finalizzata ad una convivenza sociale armoniosa e allo sviluppo della scienza, della tecnologia e dell'innovazione. Ciò che appare piuttosto caratteristico e a tratti singolare è la scelta del costituente di individuare persino le materie sulle quali il sistema educativo è chiamato a concentrarsi, come ad esempio la geografia, la cultura, i diritti umani, le caratteristiche sociali ed economiche, i valori archeologici, storici ed artistici, linguistici e tradizionali dello stato di Querètaro e dello Stato messicano.

Infine occorre sottolineare l'attenzione particolare per il valore della cultura dei cittadini di Querètaro quale diritto fondamentale. La Costituzione rinvia alla legge il compito di proteggere il patrimonio e gli eventi culturali. Alle istituzioni, con la partecipazione responsabile dell'intera società, è attribuito il compito di salvaguardare, conservare e proteggere il patrimonio culturale, diffondendone la conoscenza.

\section{L'IMPRONTA PRESIDENZIALISTA NELL'ORGANIZZAZIONE FEDERALE}

Il Messico ha consolidato lungo l'arco di una bicentenaria storia di nazione indipendente, con l'eccezione dei due periodi di governo imperiale (182123 e 1864-67), una tradizione di governo repubblicana e presidenziale. ${ }^{7}$ Il

e Apa, Ernesto e Bassini, Marco (cur.), Diritti e libertà in Internet, Le Milano, Monnier, 2017 , pp. 47 e ss. Si tratta di una problematica che richiama fondamentalmente la questione del digital divide, sulla quale v. Nannipieri, Lorenzo, "La dimensione costituzionale del digital divide. In particolare gli ostacoli cognitivi alla proiezione dell'individuo nello spazio virtuale", in Nisticò, Michele e Passaglia, Paolo (cur.), Internet e Costituzione, Torino, Giappichelli, 2014, pp. 189 e ss.; cfr. anche Pucci, Tommaso, "Il diritto all'accesso nella società dell'informazione e della conoscenza. Il digital divide", Informatica e diritto, n. 2, 2002, pp. 121 e ss.; Zocchi, Paolo, Internet: la democrazia possibile: come vincere la sfida del digital divide, Milano, Guerini, 2003.

7 Valadés, Diego, Problemas y perspectivas del sistema presidencial mexicano, in Alcántara Sáez, Manuel e Hernández Norzagaray, Ernesto (cur.), México, el nuevo esce- 
Potere Esecutivo concentrato nelle mani di un organo monocratico caratterizza sia l'ordinamento federale sia quello statale. ${ }^{8}$ Al governatore, eletto direttamente dai cittadini di Querètaro (art. 20, Cost. stat.), spetta il compito di garantire la libertà, la pace, l'ordine pubblico, la sicurezza sociale e di assumere il comando in quei casi giudicati causa di forza maggiore o grave turbamento dell'ordine pubblico. In quanto capo del governo, il presidente della Repubblica ha anche il potere di nominare e rimuovere liberamente i dipendenti pubblici dell'Esecutivo. Tutte le cariche elettive (tra questi anche i componenti della jiunta e del Parlamento) sono incompatibili con quelle di magistrato, di ministro del culto o di componenti delle forze di polizia (art. 8, Cost. stat.).

L'attribuzione ad un organo monocratico del Potere Esecutivo e di altri poteri di così ampia portata è caratteristica che difficilmente si riscontra, in questi termini, nel contesto europeo ma, come è stato altrove evidenziato, "l'opzione per la forma di governo presidenziale, con la torsione presidenzialista, ha rappresentato il filo rosso che ha attraversato la tradizione costituzionale messicana dopo l'indipendenza, diventando uno dei cardini della costituzione materiale di questo paese senza soluzione di continuità". ${ }^{9}$ Il modello, adottato inizialmente dalla Costituzione del 1824, fu replicato da quella successiva del 1857 , per essere poi confermato definitivamente con la Costituzione del 1917. Il costituzionalismo messicano, pur nel succedersi di regimi con caratteristiche diverse, si è sviluppato sostanzialmente riconoscendo al presidente della Repubblica (e, per declinazione territoriale, ai presidenti degli Stati federati) un ruolo chiave nell'articolazione del sistema dei poteri pubblici. Le Costituzioni statali sembrano replicare per gemmazione il modello presidenziale federale.

Nel dibattito in assemblea costituente, la scelta federale sulla forma di governo fu una delle questioni più discusse. Non mancarono opinioni cri-

nario politico del bicentenario, Salamanca, Ediciones Universidad de Salamanca, 2009, pp. 67 e ss.; Carpizo, Jorge, El presidencialismo mexicano, Messico, Siglo XXI, 1998, pp. 190 e ss.

8 La Costituzione federale messicana attribuisce il Potere Esecutivo al presidente della Repubblica (art. 80), eletto dal corpo elettorale a suffragio universale e diretto (art. 81). Cfr. Olivetti, Marco, "La forma di governo - Messico", in Bifulco, Raffele (cur.), Ordinamenti federali comparati, cit., p. 164.

9 Tarchi, Rolando, "La forma di governo del Messico: dal presidenzialismo imperiale alla «parlamentarizzazione» del presidenzialismo?", DPCE-online, n. 4, 2017, p. 893, reperibile in http://www.dpceonline.it/ index.php/ dpceonline/ article/view/468, data di accesso 27 agosto 2018 . 
tiche nei riguardi del modello di presidenzialismo che si stava costruendo e che si annunciava un po' troppo accentuato. ${ }^{10}$ La scelta, in realtà, fu il frutto di una serie di ragioni concomitanti, tanto di matrice culturale che politica, che rispondevano anche ad esigenze di reazione nei confronti del vecchio ordine Costituzionale. In presenza di una società assai pluralista e, per certi versi, non in grado di esprimere una rappresentanza politica adeguata ed efficiente, il modello costituzionale doveva conformarsi alle caratteristiche della realtà messicana e conservare un potere esecutivo forte, attivo e concentrato in un organo monocratico idoneo a garantire l'unità di indirizzo politico dello Stato. ${ }^{11}$ Inoltre, un presidente dotato di poteri molto ampi si sarebbe trovato nella condizione migliore per attuare in tempi più rapidi ed in maniera più efficace gli ideali rivoluzionari ed il programma di riforme sociali promesse al popolo, delle quali egli assumeva il ruolo di garante. Proprio questo binomio tra idee fortemente conservatrici e utopie rivoluzionarie ha prodotto, in maniera più o meno consapevole, una forma di presidenzialismo definito "duro e puro". ${ }^{12}$

Il presidenzialismo messicano si inserisce in un sistema federale che si fonda sul combinato disposto degli articoli 2 e 40 della Costituzione del 1917: il primo stabilisce il principio dell'unità ed indivisibilità della nazione messicana, mentre il secondo dichiara la volontà del popolo messicano di costituirsi in una Repubblica rappresentativa, democratica, federale, composta da Stati liberi e sovrani per tutto ciò che concerne il proprio regime interno, e tuttavia uniti in una federazione. Il sistema federale e la forma di governo presidenziale si condizionano a vicenda. Come è stato autorevolmente affermato, il federalismo messicano costituisce "uno strumento utile per temperare il presidenzialismo": ${ }^{13}$ spesso offre ai partiti sconfitti a livello federale la possibilità di governare in alcuni Stati e

\footnotetext{
10 Carpizo, Jorge, Sistema presidencial mexicano: dos siglos de evolución, Perú, Adrus, 2011.

11 Tarchi, Rolando, "La forma di governo del Messico: dal presidenzialismo imperiale alla «parlamentarizzazione» del presidenzialismo?", cit., p. 892. Il presidente Venustiano Carranza, aprendo i lavori del Congresso costituente, sostenne l'esigenza di preferire il presidenzialismo al parlamentarismo, secondo modalità che dovevano limitare il potere del Congresso, così da impedire che "potesse disturbare o rendere imbarazzante e difficile l'avanzata del potere esecutivo", Carpizo, Jorge e Carbonell, Miguel, Diritto costituzionale messicano, cit., 136 e ss.
}

12 Ibidem, 139.

13 Carbonell, Miguel, "Il federalismo in Messico: principi generali e distribuzioni di competenze", Diritto Pubblico Comparato ed Europeo, n. 2, 2001, p. 502. 
ne attenua il massimalismo, riducendo così le potenzialità escludenti del presidenzialismo. $^{14}$

In questo quadro, le competenze tra Stato federale e Stati federati sono ripartite secondo un principio sostanzialmente residuale. L'art. 17 della Costituzione di Querètaro afferma che il Potere Legislativo statale si estende su tutte le materie, salvo quelle che la Costituzione federale espressamente attribuisce agli Stati Uniti Messicani. Tale principio è coerente con quanto stabilisce la stessa Costituzione federale messicana che all'art. 124 riconosce ai singoli Stati tutte le facoltà non espressamente attribuite ai “funzionari federali". L'elenco delle materie espressamente conferite alla Federazione, contenuto nell'articolo 73 della Costituzione messicana, non va tuttavia inteso in senso eccessivamente rigido o esaustivo: per quanto quasi mai applicato, ${ }^{15}$ nel sistema messicano vige infatti il principio dei poteri impliciti, in virtù del quale, al fine di assicurare la tutela del principio unitario, la Federazione può esercitare il Potere Legislativo ogni volta che ciò sia ritenuto necessario per esercitare in concreto le competenze ad essa attribuite dalla Costituzione. ${ }^{16}$ Certamente residuano in capo agli Stati la disciplina della loro organizzazione politica e quella dei rapporti tra i cittadini e lo Stato, sebbene all'interno delle opzioni imposte dalla Costituzione federale.

Si tratta di un modello che per certi versi non si discosta molto da quelli federali europei. Ad esempio nell'ordinamento federale tedesco, il criterio generale di attribuzione delle competenze legislative si basa sul principio di competenza residuale dei Länder per le materie non espressamente attribuite alla Federazione, ${ }^{17}$ a cui si affianca la possibilità per la Federazione di esercitare, oltre alla competenza esclusiva su specifiche materie previste

14 Cfr. Hernández Rodríguez, Rogelio, "La disputa por el presupuesto federal. El presidencialismo y gobiernos estatales en México", Foro Internacional, n. 1, enero-marzo, 2006, p. 104, dove si sottolinea: "el predominio del Poder Ejecutivo que por años caracterizó al sistema mexicano, no sólo se manifestaba en la subordinación del Congreso, sino también en el sometimiento de los gobiernos estatales".

15 Carpizo, Jorge e Carbonell, Miguel, Diritto costituzionale messicano, cit., p. 56

16 Nicotra, Ida, Diritto pubblico e costituzionale, Torino, Giappichelli, 2013, p. 334. Il sistema di riparto è reso piuttosto complesso a causa di un fitto sistema di criteri che distinguono le competenze "coincidenti" o concorrenti dalle competenze "coesistenti" che costituirebbero comunque una eccezione ai principi del sistema federale. Cfr. Tena Ramírez, Felipe, Derecho constitucional mexicano, Cd. de México, Porrúa, pp. 119 e ss.

17 Cfr. Art. 70, Grundgesetz. 
dalla Costituzione, ${ }^{18}$ anche i poteri che ricadono nell'ambito delle materie di competenza concorrente. ${ }^{19}$

La Costituzione di Querètaro riconosce, infine, l'importante ruolo dei municipi, che costituiscono l'elemento di base della divisione territoriale e dell'organizzazione politica e amministrativa dello stato. I singoli stati messicani, infatti, presentano una suddivisione amministrativa articolata in "liberi municipi" (art. 115, Cost. fed.) a cui sono attribuiti diversi compiti tra i quali, ad esempio, i servizi pubblici locali, la sicurezza, la viabilità, l'igiene e il decoro urbano. Altre funzioni possono essere svolte, in collaborazione con il governo federale, in materia di scuola, sanità, cultura e ambiente. La Costituzione federale non garantisce l'esistenza individuale di tutti i municipi messicani ma rinvia la loro istituzione alla competenza dei singoli stati federati.

\section{PRINCIPI DI FINANZA PUBBLICA E COSTITUZIONE ECONOMICA}

Aspetti particolarmente innovativi della Costituzione di Querètaro si rinvengono anche sul fronte della Costituzione economica, in particolare della Costituzione finanziaria ${ }^{20}$ che impone vincoli di bilancio e divieti di indebitamento a carico sia dello Stato che delle municipalità (art. 14, Cost. stat.), fatte salve le situazioni di emergenza o quelle in cui si verificano

\section{Art. 73, Grundgesetz.}

19 Art. 74, Grundgesetz. Le riforme costituzionali del 2006 e del 2008 hanno abrogato la potestà legislativa statale di emanare leggi-quadro, ai sensi dell'art. 75 del Grundgesetz. Sul tema v. Palermo, Francesco, "Il nuovo riparto delle competenze legislative nell'ordinamento federale tedesco, tra retorica ed effettività", Diritto Pubblico Comparato ed Europeo, n. 2, 2007, pp. 98 e ss.

20 Il concetto di "costituzione finanziaria" viene qui accolto nel senso descritto da Di Plinio, Giampiero, "La Costituzione economica nel processo costituente europeo", Diritto Pubblico Comparato ed Europeo, n. 4, 2003, pp. 1789 e ss., al quale si rinvia ampiamente, soprattutto: id., Diritto pubblico dell'economia, Milano, Giuffrè, 1998, dove si descrive la Costituzione finanziaria come una "partizione" della Costituzione economica che racchiude le politiche di bilancio in senso ampio (nei suoi sottoinsiemi e pertinenze, quali politiche fiscali, politiche macroeconomiche e di spesa e relative procedure, diritto contabile pubblico), e infine incentivazioni, regolazioni, iniziativa pubblica, servizi e politiche di welfare considerati sotto i profili del condizionamento finanziario e del rapporto risorse/risultati nel quadro delle politiche di stabilità e crescita. Sullo stesso tema v. anche Bassanini, Franco, "Riflessioni sulla riforma della «Costituzione finanziaria»", Quaderni Costituzionali, n. 3, 1993, pp. 423 e ss. 
precise condizioni, ossia: che le risorse siano destinate ad investimenti di lungo periodo e per l'interesse della collettività; che vi sia una espressa autorizzazione legislativa con maggioranza dei due terzi; che il debito sia sostenibile, sulla base di precisi parametri e tenuto conto delle entrate complessive. Vi è anche un richiamo alla "nazionalità" del debito come strumento strategico di protezione dell'economia messicana: è vietato, infatti, contrarre debiti con altre nazioni o con società (pubbliche o private) che si collocano fuori del territorio nazionale.

La disciplina di bilancio, dunque, si presenta piuttosto ricca di vincoli tendenzialmente oggettivi e funzionali ad evitare una eccessiva esposizione debitoria dello Stato. ${ }^{21}$ Sotto questo profilo l'esperienza messicana presenta elementi di notevole comunanza con quella europea che, come è noto, a partire dal Trattato di Maastricht, passando per il Patto di Stabilità e Crescita fino, più recentemente, al Trattato sul c.d. "Fiscal compact"22 è stata caratterizzata da trasformazioni radicali nel governo della finanza

21 Il federalismo fiscale messicano è sempre stato caratterizzato da un certo accentramento delle competenze in materia finanziaria, su cui v. ampiamente Vedaschi, Arianna, "Il federalismo fiscale messicano", in Ferrari, Giuseppe Franco (cur.), Federalismo, sistema fiscale, autonomie, Roma, Donzelli editore, 2010, pp. 499 e ss.

22 Il Fiscal compact è noto come il trattato che impone ai paesi aderenti il vincolo del pareggio di bilancio. La parte più importante del Trattato, infatti, si concentra sul cosiddetto "Patto di bilancio", ossia sul vincolo sancito dal par. 1 dell'art. 3 che prescrive, alla lett. a), che "la posizione di bilancio della pubblica amministrazione di una parte contraente è in pareggio o in avanzo". Ai sensi della lett. b), essa "si considera rispettata se il saldo strutturale annuo della pubblica amministrazione è pari all'obiettivo di medio termine specifico per il paese, quale definito nel Patto di stabilità e crescita rivisto, con il limite inferiore di un disavanzo strutturale dello $0,5 \%$ del prodotto interno lordo". Quando il rapporto tra il debito pubblico e il prodotto interno lordo è significativamente inferiore al $60 \%$ tale limite di disavanzo strutturale sale fino ad un massimo dell' $1,0 \%$ del prodotto interno lordo. Il vincolo, dunque, non è esattamente al pareggio di bilancio, quanto piuttosto ad un "equilibrio di bilancio" consistente nel rispetto di un saldo prefissato che può, entro i limiti prestabiliti, essere anche in lieve disavanzo, differente a seconda del grado di indebitamento del paese e del relativo livello di rischio. Obiettivi di bilancio più stringenti e una forcella di disavanzo più stretta $(0,5 \%)$ sono imposti ai paesi meno virtuosi sotto il profilo finanziario, Morgante, Daniele, Note in tema di "Fiscal Compact", Federalismi.it, n. 7, 2012, pp. 11 e ss., reperibile in; http://www.federalismi.it/ nv14/articolo-documento.cfm? artid=19844. Data di accesso 27-agosto-2018. Per considerazioni più analitiche, anche sotto il profilo storico e comparatistico, v. Coronidi, Francesco, "La costituzionalizzazione dei vincoli di bilancio prima e dopo il Patto Europlus", Federlismi. it, n. 5, 2012, pp. 15 e ss., reperibile in; http://www.federalismi.it/ nv14/articolo-documento.cfm? artid=19650. Data di accesso 27-agosto-2018. Cfr. anche Nugnes, Francesca, "Il Fiscal Compact. Prime riflessioni su un accordo ricognitivo", Forum di Quaderni 
pubblica, con l'introduzione di vincoli di bilancio che investono non soltanto il rapporto tra Stati membri e Unione europea ma anche tra Stati e rispettive autonomie territoriali. ${ }^{23}$ I presupposti teorici all'introduzione di tali vincoli sono rinvenibili sostanzialmente nella letteratura economica e si basano sulla circostanza che una situazione finanziaria di disequilibrio da parte di uno Stato all'interno di un ordinamento federale rischia di produrre esternalità negative nei confronti di tutti gli altri Stati. ${ }^{24}$ Le regole di costituzione finanziaria, pertanto, agganciano il livello di deficit degli Stati e, conseguentemente, i livelli di spesa pubblica, ad un elemento quantitativo, ossia il prodotto interno lordo. Ciò implica che la spesa pubblica debba essere finanziata dal sistema economico e che non sia possibile coprire il deficit mediante manovre monetarie: ${ }^{25}$ il prodotto interno lordo assurge a "misura costituzionale della correttezza finanziaria dello Stato". ${ }^{26}$

Si tratta di principi che sono rinvenibili anche nella Costituzione di Querètaro, nella misura in cui il ricorso all'indebitamento è ammesso soltanto in casi specifici, in particolare quando si tratta di spesa pubblica produttiva, ossia capace di fare crescere il prodotto interno lordo e, quindi, assicurare le condizioni per riportare la finanza pubblica in una situazione di equilibrio nei periodi successivi: "el gobierno del estado y los municipios no podrán contraer deuda pública sino cuando se destine a inversiones públicas productivas" (art. 14, Cost. stat.). ${ }^{27}$

Costituzionali, 2012, reperibile in; http://www.forumcostituzionale.it/wordpress/images/ stories/pdf/documenti_forum/ paper/0305_nugnes.pdf, data di accesso 27-agosto-2018.

23 Per una rapida disamina dei diversi aspetti coinvolti v. Fabbrini, Federico, "Il pareggio di bilancio nelle Costituzioni europee", Quaderni Costituzionali, n. 4, 2011, pp. 933 e ss.

24 Nella prospettiva europea v. Momigliano, Sandro e Zotteri, Stefania, Le regole di bilancio europee: il Patto di stabilità e crescita, Roma, Banca d'Italia, Servizio Studi, Giugno 2005, pp. 1 ss.; De Grauwe, Paul, Economia dell'integrazione monetaria, Bologna, Il Mulino, 1993; Buti, Marco e Sapir, Andrè (cur.), La politica economica nell'Unione economica e monetaria europea, Bologna, Il Mulino, 1999.

25 Come è noto, l'art. 123 vieta alla Banca centrale europea e alle banche centrali nazionali, di concedere scoperti di conto o qualsiasi altra forma di facilitazione creditizia a istituzioni, organi od organismi dell'Unione, alle amministrazioni statali, agli enti regionali, locali o altri enti pubblici, ad altri organismi di diritto pubblico o a imprese pubbliche degli Stati membri, così come l'acquisto diretto presso di essi di titoli di debito da parte della Banca centrale europea o delle banche centrali nazionali.

26 Di Plinio, Giampiero, Il common core della deregulation, Milano, Giuffrè, 2005, p. 240 .

27 Sul concetto di produttività della spesa pubblica v. ampiamente: Di Plinio, Giampiero, "Costituzione e scienza economica”, Il Politico, vol. 74, n. 3, p. 178. 
Negli ordinamenti federali, più in generale, non è infrequente la presenza di vincoli di bilancio, anche di rango costituzionale, sotto forma sia di balanced budget requirements che di tax and expenditure limitations. ${ }^{28}$ Nel sistema federale tedesco, ad esempio, la Costituzione riconosce il principio di autonomia finanziaria dei Länder affermando che ogni livello di governo deve disporre di risorse finanziare per l'assolvimento dei propri compiti e prevendendo che gli introiti di determinate imposte siano attribuiti alla Federazione e ai Länder secondo la ripartizione stabilita dall'art. 106 del Grundgesetz. Tuttavia, il processo di integrazione europea e, in particolare, l'introduzione dei vincoli di bilancio, hanno inciso sotto diversi profili sul grado di autonomia finanziaria degli Stati federati, ${ }^{29}$ specie a seguito della crisi economico-finanziaria degli ultimi anni. ${ }^{30}$ La Germania, come è noto, è stata una delle principali sostenitrici di una severa politica di bilancio in tutti i paesi dell'Eurozona. La Costituzione tedesca, infatti, è stata modificata con due successive revisioni, nel 2006 e nel 2009, proprio al fine di rafforzare le regole di coordinamento finanziario sul sistema federale tedesco e porre rimedio ai rischi di superamento dei parametri europei di stabilità. ${ }^{31}$ Le nuove norme costituzionali impongono

28 V. in dettaglio, per il modello statunitense, Di Plinio, Giampiero, "Federalismo e costituzione fiscale negli Stati Uniti d'America", in Ferrari, Giuseppe Franco (cur.), Federalismo, sistema fiscale, autonomie (modelli giuridici comparati), Roma, Donzelli editore, 2010, pp. 330 e ss.

29 Per una ricostruzione evolutiva del federalismo fiscale tedesco: Spahn, Paul Bernd e Fottinger, Wolfgang, "Germany", in Ter-Minassian, Taline, (ed.), Fiscal Federalism in Theory and Practice, Washington, IMF, 1997; Hepp, Ralf e Von Hagen, Juergen, Fiscal Federalism in Germany: Stabilization and Redistribution Before and after Unification, ZEI, CEPR Discussion Paper, N. DP7246, 2009.

30 Cfr. Ciolli, Ines, "I paesi dell'eurozona e i vincoli di bilancio, quando l'emergenza economica fa saltare gli strumenti normativi ordinari", Rivista Associazione Italiana dei Costituzionalisti, n. 1, 2012, reperibile in: http://www.rivistaaic.it/ i-paesi-dell-eurozona-e-i-vincoli-di-bilancio-quando-l-emergenza-economica-fa-saltare-gli-strumenti-normativi-ordinari.html, data di accesso 27-agosto-2018.

31 Per una analisi più compiuta del processo di riforma v. Perez, Rita, "La nuova disciplina del bilancio in Germania", Giornale di Diritto Amministrativo, n. 1, 2011, pp. 95 ss.; Bifulco, Raffaele, "Il pareggio di bilancio in Germania: una riforma costituzionale postnazionale?", Rivista Associazione Italiana dei Costituzionalisti, n. 3, 2011, pp. 1 ss., reperibile in; http://www.rivistaaic.it/ il-pareggio-di-bilancio-in-germania-una-riforma-costituzionale-postnazionale.html, data di accesso 27-agosto-2018; Pedrini, Federico, "La costituzionalizzazione tedesca del Patto europeo di stabilità: il Grundgesetz preso sul serio", Quaderni Costituzionali, n. 2, 2011, pp. 391 ss.; Bertolini, Elisa, "I rapporti finanziari intergovernativi nell' evoluzione dell'ordinamento federale tedesco", in Ferrari, 
sia al Bund che ai Länder l'obbligo all'equilibrio di bilancio derivante dai Trattati europei, ${ }^{32}$ salvo deroghe legate a particolari periodi di congiuntura negativa. In tali casi, è prevista una responsabilità congiunta per l'eventuale inottemperanza agli obblighi. Il ricorso all'indebitamento viene limitato a poche situazioni eccezionali disciplinate dal nuovo art. 115 della Costituzione tedesca, che richiede espressamente l'approvazione di una legge federale di autorizzazione e la previsione di uno specifico piano di ammortamento. In base al "Patto di stabilità nazionale" i Länder risultano corresponsabili dell'indebitamento e hanno l'obbligo di partecipare alle eventuali sanzioni stabilite dall'ordinamento europeo, secondo quanto stabilito da una apposita legge federale che dovrà avere l'approvazione del Bundesrat. Inoltre, è prevista una procedura di sorveglianza delle politiche di bilancio di Bund e Länder da attuare attraverso un apposito organo di stabilità, con l'approvazione del Bundesrat. ${ }^{33}$

Gli Stati federati accettano generalmente il condizionamento quantitativo del bilancio e addirittura lo inseriscono nelle loro Costituzioni formali, sotto forma di principio del pareggio conformemente alle teorie che si richiamano al principio del matching principle, elaborate per il decentramento fiscale negli Stati federali. ${ }^{34}$ Tali teorie affermano che, all'interno dei confini di un determinato territorio, l'allocazione ottimale delle risorse deve essere conforme ai seguenti principi: finanziamento della spesa pub-

Giuseppe Franco (cur.), Federalismo, sistema fiscale, autonomie. Modelli giuridici comparati, cit., 102 e ss.; Goldmann, Matthias, "Legarsi le mani: il quadro delle misure di riduzione del deficit in Germania", Giornale di Diritto Amministrativo, n. 12, 2010, pp. 1309 e ss.

32 Art. 109, Grundgesetz.

33 Si tratta del Consiglio di stabilità, composto dal ministro federale delle finanze, il ministro federale dell'economia e i ministri delle finanze dei Länder. Esso decide a maggioranza di due terzi con esclusione del Länder interessato. Cfr. Carboni, Giuliana Giuseppina, Federalismo fiscale comparato, Napoli, Jovene, 2013, pp. 75 e ss.

34 Il riferimento, in particolare, è alla Costituzione americana che disciplina i poteri impositivi del governo federale attribuendo al Congresso la potestà di imporre e percepire tasse, diritti, imposte e dazi, ma contestualmente anche l'obbligo di apportionment, cioè di ripartire le imposte dirette tra i vari Stati in modo proporzionale alla rispettiva popolazione. Gli Stati sono ampiamente autonomi nella scelta dei tributi e aliquote, entro poche limitazioni imposte dalla Costituzione federale, quali in particolare la riserva federale sulla tassazione sull'import/export e il divieto di ostacolare il mercato interno e le relazioni commerciali interstatali. Dal punto di vista della spesa, la maggior parte delle funzioni sono allocate a livello statale o substatale, con importanti eccezioni, tra cui la difesa nazionale, il sistema pensionistico e l'assicurazione sanitaria per anziani e disabili. 
blica attraverso risorse locali e comunque riconducibile alle fonti di entrata fiscale; pressione fiscale collegata alla responsabilità politica di fronte ai contribuenti. Si tratta, come appare subito evidente, dei corollari del "teorema del decentramento". 35

Anche la Costituzione di Querètaro sembra evocare tale principio, nel momento in cui afferma che "al menos el treinta por ciento de la contratación de deuda pública debe respaldarse con contribuciones propias del estado o municipios". In altri termini, vi è un esplicito vincolo di corresponsabilità dei territori al risanamento delle finanze pubbliche, i quali sono chiamati a concorrere alla copertura del debito pubblico con proprie risorse. Le teorie del matching principle, in effetti, si basano sulla connessione tra uscite di bilancio e fonti locali di finanziamento, tra responsabilità politica di spesa e capacità territoriale di produrre e reperire le risorse necessarie. Tali teorie si stanno affermando ormai in molti Stati federali. Il modello americano, ad esempio, realizza sia il matching principle che uno dei più bassi livelli di fiscal imbalance, in un contesto di federalismo competitivo e duale in cui il riequilibrio è in gran parte affidato al gioco spontaneo di libertà economica, mobilità dei fattori produttivi e apertura del mercato, la perequazione interstatale sostanzialmente non esiste, e l'equalization è realizzata (nei limiti in cui lo è) dalla Federazione. ${ }^{36}$ In altre federazioni, come la Germania, il matching principle è tendenziale ma lo squilibrio fiscale è molto contenuto, in un contesto di federalismo cooperativo, alta perequazione intergovernativa ed elevata spesa per il welfare. ${ }^{37}$

Gli ordinamenti basati sul modello federale tendono generalmente a legare in maniera importante le funzioni attribuite agli Stati federali con il relativo potere di spesa. La Costituzione tedesca, ad esempio, stabilisce come principio generale che le spese relative all'esercizio delle funzioni del Bund e dei Länder devono gravare in maniera separata sui rispettivi

35 Cfr. Oates, Wallace E., Fiscal Federalism, New York, Harcourt Brace Jovanovich, 1972; Bird, Richard M., "Threading the Fiscal Labyrinth: Some Issues in Fiscal Decentralization”, National Tax Journal, n. 46, vol. 2, 1993, pp. 207 e ss.; Samuelson, Paul A., "The Pure Theory of Public Expenditure", The Review of Economics and Statistics, n. 4, vol. 36, 1954, pp. 387 e ss.; Buchanan, James M. e Tullock, Gordon, The Calculus of Consent, University Michigan, Michigan Press, 1962; Musgrave, Richard A., The Theory of Public Finance, New York, McGraw-Hill, 1959.

36 Dam, Kenneth W., "The American Fiscal Constitution", University of Chicago Law Review, n. 44, 1977, pp. 271 e ss.

37 Spahn, Paul Bernd e Fottinger, Wolfgang, "Germany", in Ter-Minassian, Taline, (ed.), Fiscal Federalism in Theory and Practice, cit. 
bilanci, salvo che la stessa Costituzione non preveda diversamente. ${ }^{38} \mathrm{Co}-$ erentemente, è stato reso esplicito il principio in base al quale, qualora il Bund dovesse delegare specifiche funzioni ai Länder, occorrerà fornire a questi ultimi le risorse necessarie.

In complesso, quindi, si rinviene un "principio di separazione" che risulta determinante per l'autonomia finanziaria dei Länder, in quanto occorre che essi dispongano di garanzie circa la disponibilità di risorse proprie per ciascuna delle funzioni loro attribuite. ${ }^{39}$ Il Tribunale federale tedesco, a tal proposito, ha considerato la corrispondenza tra responsabilità della spesa ed entrata come un principio fondamentale alla base dell'autonomia: $\mathrm{Fe}$ derazione e Länder devono essere messi in condizione di avere le risorse necessarie all'assolvimento dei propri compiti. ${ }^{40}$

Il principio del matching principle è rinvenibile anche nel sistema federale belga, seppure all'interno di un assemblaggio di paradossi, ${ }^{41}$ in cui l'intreccio dei confini linguistici e dei livelli di governo, disegnato sin dagli anni ' 60 per determinare le aree a fini amministrativi, ha progressivamente assunto rilievo costituzionale, divorando funzioni e competenze al livello federale. Intorno al groviglio linguistico, economico e politico si è sedimentata nel tempo, a partire dal 1970, una varietà e di forme organizzative e procedurali frammentate e sovrapposte, il cui sbalorditivo carattere è la coesistenza su medesime porzioni di territorio di un doppio strato di istituzioni differenziate, ciascuna con propri organi "costituzionali”, apparati, competenze e bilanci. In Belgio esiste una devolution spinta e contempora-

\footnotetext{
38 Art. 104a, Grundgesetz.
}

39 Cfr. sul tema: Mezzetti, Luca, "Il sistema federale tedesco", in Gambino, Silvio (cur.), Regionalismo, federalismo, devolution, Milano, Giuffrè, 2003, pp. 199 e ss.; Carboni, Giuliana Giuseppina, Federalismo fiscale comparato, cit., p. 101.

40 Bundesverfassungsgericht, sent. 72, 330 del 24 giugno 1986. Tuttavia, si tratta di un principio non privo di eccezioni. La stessa Costituzione tedesca prevede che, in taluni casi, la spesa per una funzione possa essere sostenuta congiuntamente da più livelli o da un livello diverso da quello che esercita la funzione stessa, sicché le "aree di responsabilità finanziaria comune" risultano piuttosto numerose, Woelk, Jens, "I rapporti finanziari fra livelli di governo nell'ordinamento tedesco", in Palermo, Francesco e Nicolini, Matteo (cur.), Atti del Convegno "Federalismo fiscale in Europa: esperienze straniere e spunti per il caso italiano, Napoli, Edizioni Scientifiche Italiane, 2012, pp. 15 e ss.

41 Di Plinio, Giampiero, "L'esperienza belga di federalizzazione nell'ottica del sistema della finanza pubblica", in Ferrari, Giuseppe Franco (cur.), Federalismo, sistema fiscale, autonomie, cit., pp. 167 e ss.; Uyttendaele, Marc, Précis de droit constitutionnel belge - Regards sur un système institutionnel paradoxal, Bruxelles, Bruylant, 2006. 
neamente una sovranità fiscale accentrata. Il matching principle è costituzionalmente dichiarato come corrispondenza territoriale tra spese e entrate (just retour), ${ }^{42} \mathrm{e}$ in presenza di una dose massiva di fiscal imbalance, e di un corrispondente elevato volume di trasferimenti federali. ${ }^{43} \mathrm{Ne}$ risulta una architettura istituzionale "cubista" il cui funzionamento sarebbe un mistero, se non si tenesse in considerazione lo spiccato consociativismo legislativo e istituzionale ${ }^{44}$ che ha accelerato la federalizzazione dei poteri centrali e consentito ai "poteri linguistici" e alle istanze federaliste di installarsi stabilmente in tutti gli organi costituzionali, ${ }^{45}$ dando origine al più rilevante esempio di combinazione tra la concezione "territoriale" e la concezione "personale" di federalismo. ${ }^{46}$

Come è stato altrove efficacemente affermato:

...nella teoria giuridica del federalismo fiscale, il matching principle è norma economica di nucleo costituzionale. Come in genere avviene per tutte le norme costituzionali imposte dall'economia, essa è "direttiva" e "tendenziale", e in nessun ordinamento può dirsi compiutamente realizzata; esperienze di squilibri fiscali esistono in tutti i sistemi federali, ciascuno dei quali ha risposto compensando i problemi e le tensioni con ulteriori tecniche costituzionali che tengono conto della specifica complessità del contesto istituzionale, delle tradizioni giuridiche e costituzionali, dei tipi di conflitti che il

42 Pagano, Giuseppe, Le financement des régions et des communautés 1970-2002: solidarité, responsabilité, autonomie et concurrence fiscale, Bruxelles, Crisp, 2002, pp. 5 e ss.; Benedikter, Thomas, "Esperienze europee di federalismo fiscale e del finanziamento delle Regioni a confronto con la realtà italiana", in Pföstl, Eva (cur.), Il federalismo fiscale, Roma, Apes, 2008, p. 132.

43 Badriotti, Augusta e Fornasini, Margherita e Vaneecloo, Clément, "L'evoluzione della costituzione in Belgio e in Italia: un'analisi delle relazioni fiscali intergovernative", Rivista di Politica Economica, n. VII-VIII, 2006, pp. 229 e ss.

44 Peters, B. Guy, "Consociationalism, Corruption and Chocolate: Belgian Exceptionalism", West European Politics, n. 5, 2006, pp. 1079 e ss.; Leroy, Michel, Il federalismo belga, Istituto di Studi sui Sistemi Regionali Federali e sulle Autonomie, 2007, reperibile in: http://www.issirfa.cnr.it/ michel-leroy-il-federalismo-belga-25-6-2007.html, data di accesso 27-agosto-2018.

45 Olivetti, Marco, "Il federalismo asimmetrico belga e le sue recenti evoluzioni", in D'Ignazio, Guerino (cur.), Integrazione europea e asimmetrie regionali: modelli a confronto, Milano, Giuffrè, 2007, pp. 63 e ss.

46 Spahn, Paul Bernd, "Managing Fiscal Conflicts", in Watts, Ronald L. e Chattopadhyay, Rupak (eds.), Unity in Diversity: Learning from Each Other, vol. 2, Emerging Issues in Fiscal Federalism, New Delhi, Viva Books, 2008, p. 53. 
federalismo fiscale genera (esclusivamente territoriali, o culturali/linguistici, o socioeconomici, etc.). ${ }^{47}$

L'attenzione della Costituzione di Querètaro alle regole di finanza pubblica appare, in sostanza, un elemento di notevole modernità che si inserisce in un filone ormai comune negli ordinamenti federali occidentali. I principi di finanza pubblica sana e di produttività della spesa appaiono coerenti con le più diffuse teorie giuridiche ed economiche che mirano a garantire la sostenibilità delle politiche sociali e la tutela intergenerazionale dei diritti. A ciò si aggiunge anche una visione realistica dei "vizi" e delle virtù dei decisori pubblici che emerge, ad esempio, quando la Costituzione di Querètaro chiude il sistema di regole di costituzione finanziaria affermando il principio in base al quale nessun governo è autorizzato a contrarre debiti durante l'ultimo anno della legislatura. Si tratta di un chiaro esempio del tentativo di limitare gli effetti della cosiddetta c.d. teoria economica del ciclo elettorale della spesa pubblica. L'obiettivo, evidentemente, è quello di scongiurare il fenomeno assai diffuso in base al quale gli ultimi mesi di legislatura generalmente coincidono con un aumento consistente della spesa pubblica finanziato con il ricorso al deficit al solo scopo di rafforzare il consenso popolare in vista delle elezioni, senza badare alle conseguenze negative che ricadono su cui sarà chiamato a governare il bilancio pubblico negli anni successivi.

Un principio costituzionale di questo tipo farebbe assai bene anche ai paesi europei dove il continuo tentativo, da parte dei governi nazionali, di violare o considerare in maniera più "elastica" $i$ vincoli di bilancio imposti dall'Unione Europea, specie in periodi “caldi" dal punto di vista elettorale, rappresenta uno dei motivi di conflitto più frequenti tra istituzioni statali ed europee.

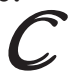

47 Di Plinio, Giampiero, Costituzione e scienza economica, cit., 153 e ss.; Bird, Richard M. e Tarasov, Andrey V., "Closing the gap: fiscal imbalances and intergovernmental transfers in developed federations", in Environment \& Planning, n. 12004 , pp. 77 e ss. 